\title{
GRADED BETTI NUMBERS OF PATH IDEALS OF CYCLES AND LINES
}

\author{
ALI ALILOOEE AND SARA FARIDI
}

\begin{abstract}
We use purely combinatorial arguments to give a formula to compute all graded Betti numbers of path ideals of paths and cycles. As a consequence we can give new and short proofs for the known formulas of regularity and projective dimensions of path ideals of paths.
\end{abstract}

\section{INTRODUCTION}

Path complexes are simplicial complexes whose facets encode paths of a fixed length in a graph. These simplicial complexes in turn correspond to monomial ideals called "path ideals". Path ideals of graphs were first introduced by [CN99] in a different algebraic context, but the study of algebraic invariants corresponding to their minimal free resolutions has become popular, with works of [BHK10] and [HT10], and the authors AF15]. The papers cited above give partial information on Betti numbers of path ideals. In this paper we use purely combinatorial arguments based on our results in [AF15] to give an explicit formula for all the graded Betti numbers of path ideals of paths and cycles. As a consequence we can give new and short proofs for the known formulas of regularity and projective dimensions of path ideals of path graphs.

\section{Preliminaries}

A simplicial complex on vertex set $\mathcal{X}=\left\{x_{1}, \ldots, x_{n}\right\}$ is a collection $\Delta$ of subsets of $\mathcal{X}$ such that $\left\{x_{i}\right\} \in \Delta$ for all i, and if $F \in \Delta$ and $G \subset F$, then $G \in \Delta$. The elements of $\Delta$ are called faces of $\Delta$ and the maximal faces under inclusion are called facets of $\Delta$. We denote the simplicial complex $\Delta$ with facets $F_{1}, \ldots, F_{s}$ by $\left\langle F_{1}, \ldots, F_{s}\right\rangle$. We call $\left\{F_{1}, \ldots, F_{s}\right\}$ the facet set of $\Delta$ and is denoted by $F(\Delta)$. The set $\mathcal{X}$ is the vertex set of $\Delta$ and is denoted by $\operatorname{Vert}(\Delta)$. A subcollection of $\Delta$ is a simplicial complex whose facet set is a subset of the facet set of $\Delta$. For $\mathcal{Y} \subseteq \mathcal{X}$, an induced subcollection of $\Delta$ on $\mathcal{Y}$, denoted by $\Delta \mathcal{Y}$, is the simplicial complex whose vertex set is a subset of $\mathcal{Y}$ and facet set is $\{F \in F(\Delta) \mid F \subseteq \mathcal{Y}\}$. If $F$ is a face of $\Delta=\left\langle F_{1}, \ldots, F_{s}\right\rangle$ and $\mathcal{X}=\operatorname{Vert}(\Delta)$, the complements of a facet $F_{i}$ and of the simplicial complex $\Delta$ are

$$
\left(F_{i}\right)_{\mathcal{X}}^{c}=\mathcal{X} \backslash F_{i} \text { and } \Delta_{\mathcal{X}}^{c}=\left\langle\left(F_{1}\right)_{\mathcal{X}}^{c}, \ldots,\left(F_{s}\right)_{\mathcal{X}}^{c}\right\rangle .
$$

Note that if $\mathcal{Y} \varsubsetneqq \operatorname{Vert}(\Delta)$, then $\left(\Delta_{\mathcal{X}}^{c}\right)_{\mathcal{Y}}=\left(\Delta_{\mathcal{Y}}\right)_{\mathcal{Y}}^{c}$.

From now on we assume that $R=K\left[x_{1}, \ldots, x_{n}\right]$ is a polynomial ring over a field $K$. Suppose that $I$ is an ideal in $R$ minimally generated by square-free monomials $M_{1}, \ldots, M_{s}$. The facet complex $\Delta(I)$ associated to $I$ has vertex set $\left\{x_{1}, \ldots, x_{n}\right\}$ and is defined as $\Delta(I)=\left\langle F_{1}, \ldots, F_{s}\right\rangle$ where $F_{i}=\left\{x_{j}\left|x_{j}\right| M_{i}, 1 \leq j \leq n\right\}, 1 \leq i \leq s$. Conversely if $\Delta$ 
is a simplicial complex with vertices labeled $x_{1}, \ldots, x_{n}$, the facet ideal of $\Delta$ is defined as $I(\Delta)=\left(\prod_{x \in F} x \mid F\right.$ is a facet of $\left.\Delta\right)$.

Given a homogeneous ideal $I$ of the polynomial ring $R$ there exists a graded minimal finite free resolution

$$
0 \rightarrow \bigoplus_{d} R(-d)^{\beta_{p, d}} \rightarrow \cdots \rightarrow \bigoplus_{d} R(-d)^{\beta_{1, d}} \rightarrow R \rightarrow R / I \rightarrow 0
$$

of $R / I$ in which $R(-d)$ denotes the graded free module obtained by shifting the degrees of elements in $R$ by $d$. The numbers $\beta_{i, d}$ are the $i$-th $\mathbb{N}$-graded Betti numbers of degree $d$ of $R / I$, and are independent of the choice of graded minimal finite free resolution.

By a reformulation of Hochster's formula in [AF15, Theorem 2.8], to compute Betti numbers we only need to consider induced subcollections $\Gamma=\Delta \mathcal{Y}$ of a simplicial complex $\Delta$ with $\mathcal{Y}=\operatorname{Vert}(\Gamma)$.

\section{Path COMplexes AND RUnS}

Definition 3.1. Let $G=(\mathcal{X}, E)$ be a finite simple graph and let $t$ be an integer such that $t \geq 2$. If $x$ and $y$ are two vertices of $G$, a path (or path) of length $(t-1)$ from $x$ to $y$ is a sequence of distinct vertices $x=x_{i_{1}}, \ldots, x_{i_{t}}=y$ of $G$ such that $\left\{x_{i_{j}}, x_{i_{j+1}}\right\} \in E$ for all $j=1,2, \ldots, t-1$. We define the path ideal of $G$, denoted by $I_{t}(G)$ to be the ideal of $K\left[x_{1}, \ldots, x_{n}\right]$ generated by the monomials of the form $x_{i_{1}} x_{i_{2}} \ldots x_{i_{t}}$ where $x_{i_{1}}, x_{i_{2}}, \ldots, x_{i_{t}}$ is a path in $G$. The facet complex of $I_{t}(G)$, denoted by $\Delta_{t}(G)$, is called the path complex of the graph $G$.

Two special cases that we will be considering in this paper are when $G$ is a cycle $C_{n}$, or a path graph (or path) $L_{n}$ on vertices $\left\{x_{1}, \ldots, x_{n}\right\}$.

$$
C_{n}=\left\langle x_{1} x_{2}, \ldots, x_{n-1} x_{n}, x_{n} x_{1}\right\rangle \text { and } L_{n}=\left\langle x_{1} x_{2}, \ldots, x_{n-1} x_{n}\right\rangle .
$$

Example 3.2. Consider the cycle $C_{5}$ with vertex set $\mathcal{X}=\left\{x_{1}, \ldots, x_{5}\right\}$ Then

$$
I_{4}\left(C_{5}\right)=\left(x_{1} x_{2} x_{3} x_{4}, x_{2} x_{3} x_{4} x_{5}, x_{3} x_{4} x_{5} x_{1}, x_{4} x_{5} x_{1} x_{2}, x_{5} x_{1} x_{2} x_{3}\right) .
$$

Notation 3.3. Let $i$ and $n$ be two positive integers. For (a set of) labeled objects we use the notation $\bmod n$ to denote

$$
x_{i} \bmod n=\left\{x_{j} \mid 1 \leq j \leq n, i \equiv j \bmod n\right\}
$$

and

$$
\left\{x_{u_{1}}, x_{u_{2}}, \ldots, x_{u_{t}}\right\} \quad \bmod n=\left\{x_{u_{j}} \bmod n \mid j=1,2, \ldots, n\right\} .
$$

Let $C_{n}$ be a cycle on vertex set $\mathcal{X}=\left\{x_{1}, \ldots, x_{n}\right\}$ and $t<n$. The standard labeling of the facets of $\Delta_{t}\left(C_{n}\right)$ is as follows.

We let $\Delta_{t}\left(C_{n}\right)=\left\langle F_{1}, \ldots, F_{n}\right\rangle$ where $F_{i}=\left\{x_{i}, x_{i+1}, \ldots, x_{i+t-1}\right\} \bmod n$ for all $1 \leq i \leq n$. Since for each $1 \leq i \leq n$ we have

$$
F_{i+1} \backslash F_{i}=\left\{x_{t+i}\right\} \text { and } F_{i} \backslash F_{i+1}=\left\{x_{i}\right\} \quad \bmod n,
$$

it follows that $\left|F_{i} \backslash F_{i+1}\right|=1$ and $\left|F_{i+1} \backslash F_{i}\right|=1 \quad \bmod n$ for all $1 \leq i \leq n-1$.

Definition 3.4. Given an integer $t$, we define a run to be the path complex of a path graph. A run which has $p$ facets is called a run of length $p$ and corresponds to $\Delta_{t}\left(L_{p+t-1}\right)$. Therefore, a run of length $p$ has $p+t-1$ vertices. 
Example 3.5. Consider the cycle $C_{7}$ on vertex set $\mathcal{X}=\left\{x_{1}, \ldots x_{7}\right\}$ and the simplicial complex $\Delta_{4}\left(C_{7}\right)$. The following induced subcollections are two runs in $\Delta_{4}\left(C_{7}\right)$

$$
\begin{aligned}
& \Delta_{1}=\left\langle\left\{x_{1}, x_{2}, x_{3}, x_{4}\right\},\left\{x_{2}, x_{3}, x_{4}, x_{5}\right\}\right\rangle \\
& \Delta_{2}=\left\langle\left\{x_{1}, x_{2}, x_{6}, x_{7}\right\},\left\{x_{1}, x_{2}, x_{3}, x_{7}\right\},\left\{x_{1}, x_{2}, x_{3}, x_{4}\right\}\right\rangle .
\end{aligned}
$$

In AF15] we show that every induced subcollection of the path complex of a cycle is a disjoint union of runs [AF15, Proposition 3.6], and that two induced subcollections of the path complex of a cycle composed of the same number of runs of the same lengths are homeomorphic Ali15, Lemma 3.2.9]. Then all the information we need to compute the homologies of induced subcollections of $\Delta_{t}\left(C_{n}\right)$ depends on the number and the lengths of the runs.

Definition 3.6. For a fixed integer $t \geq 2$, let the pure $(t-1)$-dimensional simplicial complex $\Gamma=\left\langle F_{1}, \ldots, F_{s}\right\rangle$ be a disjoint union of runs of length $s_{1}, \ldots, s_{r}$. Then the sequence of positive integers $s_{1}, \ldots, s_{r}$ is called a run sequence on $\mathcal{Y}=\operatorname{Vert}(\Gamma)$, and we use the notation

$$
E\left(s_{1}, \ldots, s_{r}\right)=\Gamma_{\mathcal{Y}}^{c}=\left\langle\left(F_{1}\right)_{\mathcal{Y}}^{c}, \ldots,\left(F_{s}\right)_{\mathcal{Y}}^{c}\right\rangle
$$

\section{Graded Betti numbers of Path ideals}

We focus on Betti numbers of degree less than $n$, as those of degree $n$ were computed in [AF15]. By [AF15, Theorem 2.8] we need to count induced subcollections.

Definition 4.1. Let $i$ and $j$ be positive integers. We call an induced subcollection $\Gamma$ of $\Delta_{t}\left(C_{n}\right)$ an $(i, j)$-eligible subcollection of $\Delta_{t}\left(C_{n}\right)$ if $\Gamma$ is composed of disjoint runs of lengths

$$
(t+1) p_{1}+1, \ldots,(t+1) p_{\alpha}+1,(t+1) q_{1}+2, \ldots,(t+1) q_{\beta}+2
$$

for nonnegative integers $\alpha, \beta, p_{1}, p_{2}, \ldots, p_{\alpha}, q_{1}, q_{2}, \ldots, q_{\beta}$, which satisfy the following conditions

$$
\begin{aligned}
& j=(t+1)(P+Q)+t(\alpha+\beta)+\beta \\
& i=2(P+Q)+2 \beta+\alpha,
\end{aligned}
$$

where $P=\sum_{i=1}^{\alpha} p_{i}$ and $Q=\sum_{i=1}^{\beta} q_{i}$.

Eligible subcollections count the graded Betti numbers.

Theorem 4.2 ( AF15] Theorem 5.3). Let $I=I(\Lambda)$ be the facet ideal of an induced subcollection $\Lambda$ of $\Delta_{t}\left(C_{n}\right)$. Suppose that $i$ and $j$ are integers with $i \leq j<n$. Then the $\mathbb{N}$-graded Betti number $\beta_{i, j}(R / I)$ is the number of $(i, j)$-eligible subcollections of $\Lambda$.

The following corollary is a special case of Theorem 4.2.

Corollary 4.3. Let $I=I(\Lambda)$ be the facet ideal of an induced subcollection $\Lambda$ of $\Delta_{t}\left(C_{n}\right)$. Then for every $i, \beta_{i, t i}(R / I)$, is the number of induced subcollections of $\Lambda$ which are composed of $i$ runs of length 1 .

Proof. From Theorem 4.2 we have $\beta_{i, t i}(R / I)$ is the number of $(i, t i)$-eligible subcollections of $\Lambda$. With notation as in Definition 4.1 we have

$$
\left\{\begin{array}{l}
t i=(t+1)(P+Q)+t(\alpha+\beta)+\beta \\
i=2(P+Q)+(\alpha+\beta)+\beta
\end{array} \Rightarrow t i=2 t(P+Q)+t(\alpha+\beta)+t \beta\right.
$$


Putting the two equations for $t i$ together, we conclude that $(t-1)(P+Q+\beta)=0$. But $\beta$, $P, Q \geq 0$ and $t \geq 2$, so we must have

$$
\beta=P=Q=0 \Rightarrow p_{1}=p_{2}=\cdots=p_{\alpha}=0 .
$$

So $\alpha=i$ and $\Gamma$ is composed of $i$ runs of length one.

Theorem 4.2 holds in particular for $\Lambda=\Delta_{t}\left(L_{m}\right)$ and $\Lambda=\Delta_{t}\left(C_{n}\right)$ for any integers $m, n$. Our next statement is in a sense a converse to Theorem 4.2 .

Proposition 4.4. Let $t$ and $n$ be integers such that $2 \leq t \leq n$ and $I=I(\Lambda)$ be the facet ideal of $\Lambda$ where $\Lambda$ is an induced subcollection of $\Delta_{t}\left(C_{n}\right)$. Then for each $i, j \in \mathbb{N}$, if $i<n$ and $\beta_{i, j}(R / I) \neq 0$, there exist nonnegative integers $\ell, d$ such that $i \leq d<n$ and

$$
\left\{\begin{array}{l}
i=\ell+d \\
j=t \ell+d
\end{array}\right.
$$

Proof. From Theorem 4.2 we know $\beta_{i, j}$ is equal to the number of $(i, j)$-eligible subcollections of $\Lambda$, where with notation as in Definition 4.1 we have

$$
\left\{\begin{array}{l}
j=(t+1)(P+Q)+t(\alpha+\beta)+\beta \\
i=2(P+Q)+(\alpha+\beta)+\beta
\end{array}\right.
$$

It follows that

$$
j-i=(t-1)(P+Q+\alpha+\beta) \text { and } t i-j=(t-1)(P+Q+\beta) .
$$

We now show that there exist positive integers $\ell, d$ such that $i=\ell+d$ and $j=t \ell+d$.

$$
\left\{\begin{array}{l}
i=\ell+d \\
j=t \ell+d
\end{array} \Rightarrow \ell=\frac{j-i}{t-1} \text { and } d=\frac{t i-j}{t-1} .\right.
$$

From (2) we can see that $i$ and $j$ as described above are nonnegative integers.

Corollary 4.3 tells us that to compute Betti numbers $\beta_{i, t i}$ of induced subcollections of $\Delta_{t}\left(C_{n}\right)$ we need to count the number of its induced subcollections which consist of disjoint runs of length one. The next few pages are dedicated to counting such subcollections. We use some combinatorial methods to generalize a helpful formula which can be found in Stanley's book [Sta12, page 73].

Lemma 4.5. Consider a collection of $n$ points arranged on a line. The number of ways to color $k$ points, when there are at least $t$ uncolored points on the line between each colored point is

$$
\left(\begin{array}{c}
n-(k-1) t \\
k
\end{array}\right)
$$

Proof. First label the points by $1,2, \ldots, n$ from left to right, and let $a_{1}<a_{2}<\cdots<a_{k}$ be the colored points. For $1 \leq i \leq k-1$, we define $x_{i}$ to be the number of points, including $a_{i}$, which are between $a_{i}$ and $a_{i+1}$, and $x_{0}$ to be the number of points which exist before $a_{1}$, and $x_{k}$ the number of points, including $a_{k}$, which are after $a_{k}$.

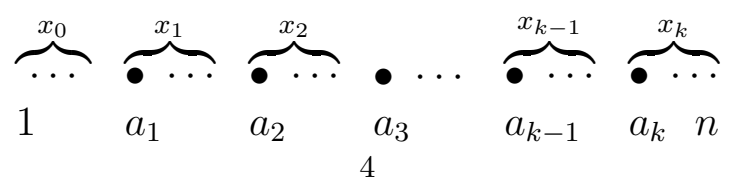


If we consider the sequence $x_{0}, x_{1}, \ldots, x_{k}$ it is not difficult to see that there is a one to one correspondence between the positive integer solutions of the following equation and the ways of coloring $k$ points of $n$ points on a line with at least $t$ uncolored points between each two colored points.

$$
x_{0}+x_{1}+\cdots+x_{k}=n \quad x_{0} \geq 0, x_{i}>t, \text { for } 1 \leq i \leq k-1, \text { and } x_{k} \geq 1 .
$$

So we only need to find the number of positive integer solutions of this equation. Consider the following equation

$$
\left(x_{0}+1\right)+\left(x_{1}-t\right)+\cdots+\left(x_{k-1}-t\right)+x_{k}=n-(k-1) t+1
$$

where $x_{0}+1 \geq 1, x_{i}-t \geq 1$, for $i=0 \ldots, k-1$ and $x_{k} \geq 1$. The number of positive integer solution of this equation is (see for example [Gri93] page 29)

$$
\left(\begin{array}{c}
n-(k-1) t \\
k
\end{array}\right)
$$

Corollary 4.6. Let $C_{n}$ be a graph cycle and with the standard labeling let $\Gamma$ be a proper subcollection of $\Delta_{t}\left(C_{n}\right)$ with $k$ facets $F_{a}, \ldots, F_{a+k-1} \bmod n$. The number of induced subcollections of $\Gamma$ which are composed of $m$ runs of length one is

$$
\left(\begin{array}{c}
k-(m-1) t \\
m
\end{array}\right) .
$$

Proof. To compute the number of induced subcollections of $\Gamma$ which are composed of $m$ runs of length one, it is enough to consider the facets $F_{a}, \ldots, F_{a+k-1}$ as points arranged on a line and compute the number of ways which we can color $m$ points of these $k$ arranged points with at least $t$ uncolored points between each two consecutive colored points. Therefore, by Lemma 4.5 we have the number of induced subcollections of $\Gamma$ which are composed of $m$ runs of length one is $\left(\begin{array}{c}k-(m-1) t \\ m\end{array}\right)$.

Proposition 4.7. Let $C_{n}$ be a graph cycle with vertex set $\mathcal{X}=\left\{x_{1}, \ldots, x_{n}\right\}$. The number of induced subcollections of $\Delta_{t}\left(C_{n}\right)$ which are composed of $m$ runs of length one is

$$
\frac{n}{n-m t}\left(\begin{array}{c}
n-m t \\
m
\end{array}\right) \text {. }
$$

Proof. Recall that $\Delta_{t}\left(C_{n}\right)=\left\langle F_{1}, \ldots, F_{n}\right\rangle$ with standard labeling. First we compute the number of induced subcollections of $\Delta_{t}\left(C_{n}\right)$ which consist of $m$ runs of length one and do not contain the vertex $x_{n}$. There are $t$ facets of $\Delta_{t}\left(C_{n}\right)$ which contain $x_{n}$, the remaining facets are $F_{1}, \ldots, F_{n-t}$, and so by Corollary 4.6 the number we are looking for is

$$
\left(\begin{array}{c}
n-t-(m-1) t \\
m
\end{array}\right)=\left(\begin{array}{c}
n-m t \\
m
\end{array}\right)
$$

Now we are going to compute the number of induced subcollections $\Gamma$ which consist of $m$ runs of length one and include $x_{n}$. We have $t$ facets which contain $x_{n}$, they are $F_{n-t+1} \ldots, F_{n}$. Each such $\Gamma$ will contain one $F_{i} \in\left\{F_{n-t+1} \ldots, F_{n}\right\}$ as the run containing $x_{n}$, and $m-1$ other runs of length one which have to be chosen so that they are disjoint from $F_{i}$. So we are 
looking for $m-1$ runs of length one in the subcollection $\Gamma^{\prime}=\left\langle F_{i+t}, \ldots, F_{i-t}\right\rangle \bmod n$. The subcollection $\Gamma^{\prime}$ has $n-2 t-1$ facets, so by Corollary 4.6 it has

$$
\left(\begin{array}{c}
n-2 t-1-(m-2) t \\
m-1
\end{array}\right)=\left(\begin{array}{c}
n-m t-1 \\
m-1
\end{array}\right)
$$

induced subcollections that consist of runs of length one. Putting this together with the number of ways to choose $F_{i}$ and with (3) we conclude that the number of induced subcollections of $\Delta_{t}\left(C_{n}\right)$ which are composed of $m$ runs of length one is

$$
t\left(\begin{array}{c}
n-m t-1 \\
m-1
\end{array}\right)+\left(\begin{array}{c}
n-m t \\
m
\end{array}\right)=\frac{n}{n-m t}\left(\begin{array}{c}
n-m t \\
m
\end{array}\right) .
$$

We apply these counting facts to find Betti numbers in specific degrees.

Corollary 4.8. Let $n \geq 2$ and $t$ be an integer such that $2 \leq t \leq n$. Then for the cycle $C_{n}$ we have

$$
\beta_{i, t i}\left(R / I_{t}\left(C_{n}\right)\right)=\frac{n}{n-t i}\left(\begin{array}{c}
n-t i \\
i
\end{array}\right) .
$$

Proof. From Corollary 4.3 we have $\beta_{i, t i}\left(R / I_{t}\left(C_{n}\right)\right)$ in each of the three cases is the number of induced subcollections of $\Delta_{t}\left(C_{n}\right)$ which are composed of $i$ runs of length 1 . The formula now follows from Proposition 4.7.

The following Lemma is the core of our counting later on in this section.

Lemma 4.9. Let $\Delta_{t}\left(C_{n}\right)=\left\langle F_{1}, F_{2}, \ldots, F_{n}\right\rangle, 2 \leq t \leq n$, be the standard labeling of the path complex of a cycle $C_{n}$ on vertex set $\mathcal{X}=\left\{x_{1}, \ldots, x_{n}\right\}$. Let $i$ be a positive integer and $\Gamma=\left\langle F_{c_{1}}, F_{c_{2}}, \ldots, F_{c_{i}}\right\rangle$ be an induced subcollection of $\Delta_{t}\left(C_{n}\right)$ consisting of $i$ runs of length 1, with $1 \leq c_{1}<c_{2}<\cdots<c_{i} \leq n$. Suppose that $\Sigma$ is the induced subcollection on $\operatorname{Vert}(\Gamma) \cup\left\{x_{c_{u}+t}\right\}$ for some $1 \leq u \leq i$. Then

$$
|\Sigma|=\left\{\begin{array}{llll}
|\Gamma|+t & u<i & \text { and } & c_{u+1}=c_{u}+t+1 \\
|\Gamma|+1 & u=i & \text { or } & c_{u+1}>c_{u}+t+1
\end{array}\right.
$$

Proof. Since $\Gamma$ consists of runs of length one and each $F_{c_{u}}=\left\{x_{c_{u}}, x_{c_{u}+1}, \ldots, x_{c_{u}+t-1}\right\}$ we must have $c_{u+1}>c_{u}+t \bmod n$ for $u \in\{1,2, \ldots, i-1\}$. There are two ways that $x_{c_{u}+t}$ could add facets to $\Gamma$ to obtain $\Sigma$.

(1) If $c_{u+1}=c_{u}+t+1$ then $F_{c_{u}}, F_{c_{u}+1}, \ldots, F_{c_{u}+t+1}=F_{c_{u+1}} \in \Sigma$ or in other words, we have added $t$ new facets to $\Gamma$.

(2) If $c_{u+1}>c_{u}+t+1$ or $u=i$ then $F_{c_{u}+1} \in \Sigma$, and therefore one new facet is added to $\Gamma$.

The following propositions, which generalize Lemma 7.4.22 in [Jac04, will help us to compute the remaining Betti numbers.

Proposition 4.10. Let $\Delta_{t}\left(C_{n}\right)=\left\langle F_{1}, F_{2}, \ldots, F_{n}\right\rangle, 2 \leq t \leq n$, be the standard labeling of the path complex of a cycle $C_{n}$ on vertex set $\mathcal{X}=\left\{x_{1}, \ldots, x_{n}\right\}$. Also let $i, j$ be positive integers such that $j \leq i$ and $\Gamma=\left\langle F_{c_{1}}, F_{c_{2}}, \ldots, F_{c_{i}}\right\rangle$ be an induced subcollection of $\Delta_{t}\left(C_{n}\right)$ consisting of $i$ runs of length 1 , with $1 \leq c_{1}<c_{2}<\cdots<c_{i} \leq n$. Suppose that $W=\operatorname{Vert}(\Gamma) \cup A \subsetneq \mathcal{X}$ 
for some subset $A$ of $\left\{x_{c_{1}+t}, \ldots, x_{c_{i}+t}\right\} \bmod n$ with $|A|=j$. Then the induced subcollection $\Sigma$ of $\Delta_{t}\left(C_{n}\right)$ on $W$ is an $(i+j, t i+j)$-eligible subcollection.

Proof. Since $\Gamma$ consists of runs of length one and each $F_{c_{u}}=\left\{x_{c_{u}}, x_{c_{u}+1}, \ldots, x_{c_{u}+t-1}\right\}$ we must have $c_{u+1}>c_{u}+t \bmod n$ for $u \in\{1,2, \ldots, i-1\}$. The runs (or connected components) of $\Sigma$ are of the form $\Sigma^{\prime}=\Sigma_{U}$ where $U \subseteq W$, and can have one of the following possible forms.

(a) For some $a \leq i$ :

$$
U=F_{c_{a}},
$$

and therefore $\Sigma^{\prime}=\left\langle F_{c_{a}}\right\rangle$ is a run of length 1 .

(b) For some $a \leq i$ :

$$
U=F_{c_{a}} \cup\left\{x_{c_{a}+t}\right\},
$$

and therefore $c_{a+1}>c_{a}+t+1$, so from Lemma 4.9 we have $\Sigma^{\prime}=\left\langle F_{c_{a}}, F_{c_{a}+1}\right\rangle$ is a run of length 2 .

(c) For some $a \leq i$ :

$$
U=F_{c_{a}} \cup F_{c_{a+1}} \cup \cdots \cup F_{c_{a+r}} \cup\left\{x_{c_{a}+t}, x_{c_{a+1}+t}, \ldots, x_{c_{a+r-1}+t}\right\} \quad \bmod n
$$

and $F_{c_{a+j}}=F_{c_{a}+j(t+1)}$ for $j=0,1, \ldots, r$ and $r \geq 1$. Then from Lemma 4.9 above we know $\Sigma^{\prime}$ is a run of length $r+1+t r=(t+1) r+1$.

(d) For some $a \leq i$ :

$$
U=F_{c_{a}} \cup F_{c_{a+1}} \cup \cdots \cup F_{c_{a+r}} \cup\left\{x_{c_{a}+t}, x_{c_{a+1}+t}, \ldots, x_{c_{a+r}+t}\right\} \quad \bmod n
$$

and $F_{c_{a+j}}=F_{c_{a}+j(t+1)}$ for $j=0,1, \ldots, r$ and $r \geq 1$, and $c_{a+r+1}>c_{a+r}+t+1$ or $a+r=i$. Then from Lemma 4.9 we have $\Sigma^{\prime}$ is a run of length $r+1+t r+1=$ $(t+1) r+2$.

So we have shown that $\Sigma$ consists of runs of length 1 and $2 \bmod t+1$.

Suppose that the runs in $\Sigma$ are of the form described in (1). By Definition 3.4 we have

$$
\begin{aligned}
|\operatorname{Vert}(\Sigma)| & =(t+1) p_{1}+t+\cdots+(t+1) p_{\alpha}+t+(t+1) q_{1}+t+1+\cdots+(t+1) q_{\beta}+t+1 \\
& =(t+1) P+t \alpha+(t+1) Q+t \beta+\beta \\
& =(t+1)(P+Q)+t(\alpha+\beta)+\beta .
\end{aligned}
$$

On the other hand by the definition of $\Sigma$ we know that, $\Sigma$ has $t i+j$ vertices and therefore

$$
t i+j=(t+1)(P+Q)+t(\alpha+\beta)+\beta .
$$

It remains to show that $i+j=2(P+Q)+(\alpha+\beta)+\beta$. Note that if $j=0$ then $\beta=P=Q=0$ and hence

$$
j=0 \Longrightarrow P+Q+\beta=0 .
$$

Moreover each vertex $x_{c_{v}+t} \in A$ either increases the length of a run in $\Gamma$ by one and hence increases $\beta$ (the number of runs of length 2 in $\Gamma$ ) by one, or increases the length of a run by $t+1$, in which case $P+Q$ increases by 1 . We can conclude that if we add $j$ vertices to $\Gamma, P+Q+\beta$ increases by $j$. From this and (4) we have $j=P+Q+\beta$. Now we solve the following system

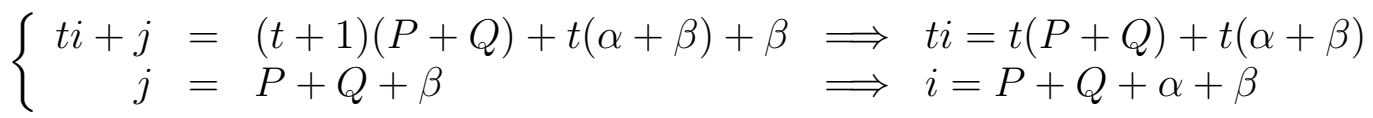




$$
\Longrightarrow\left\{\begin{array}{l}
i=P+Q+\alpha+\beta \\
j=P+Q+\beta
\end{array} \Longrightarrow i+j=2(P+Q)+(\alpha+\beta)+\beta\right.
$$

Proposition 4.11. Let $C_{n}$ be a cycle, $2 \leq t \leq n$, and $i$ and $j$ be positive integers. Suppose that $\Sigma$ is an $(i+j, t i+j)$-eligible subcollection of $\Delta_{t}\left(C_{n}\right), 2 \leq t \leq n$. Then with notation as in Definition 4.1, there exists a unique induced subcollection $\Gamma$ of $\Delta_{t}\left(C_{n}\right)$ of the form $\left\langle F_{c_{1}}, F_{c_{2}}, \ldots, F_{c_{i}}\right\rangle$ with $1 \leq c_{1}<c_{2}<\cdots<c_{i} \leq n$ consisting of $i$ runs of length 1 , and a subset $A$ of $\left\{x_{c_{1}+t}, \ldots, x_{c_{i}+t}\right\} \bmod n$, with $|A|=j$ such that $\Sigma=\Delta_{t}\left(C_{n}\right)_{W}$ where $W=\operatorname{Vert}(\Gamma) \cup A$.

Moreover if $\mathcal{R}=\left\langle F_{h}, F_{h+1}, \ldots, F_{h+m}\right\rangle \bmod n$ is a run in $\Sigma$ with $|\mathcal{R}|=2 \bmod (t+1)$, then $F_{h+m} \notin \Gamma \bmod n$.

Proof. Suppose that $\Sigma$ consists of runs $R_{1}^{\prime}, R_{2}^{\prime}, \ldots, R_{\alpha+\beta}^{\prime}$ where for $k=1,2, \ldots, \alpha+\beta$

$$
\begin{array}{ll}
R_{k}^{\prime}=\left\langle F_{h_{k}}, F_{h_{k}+1}, \ldots, F_{h_{k}+m_{k}-1}\right\rangle & \bmod n \\
\operatorname{Vert}\left(R_{k}^{\prime}\right)=\left\{x_{h_{k}}, x_{h_{k}+1}, \ldots, x_{h_{k}+m_{k}+t-2}\right\} & \bmod n \\
h_{k+1} \geq t+h_{k}+m_{k} & \bmod n
\end{array}
$$

and

$$
m_{k}= \begin{cases}(t+1) p_{k}+1 & \text { for } \quad k=1,2, \ldots, \alpha \\ (t+1) q_{k-\alpha}+2 & \text { for } \quad k=\alpha+1, \alpha+2, \ldots, \alpha+\beta .\end{cases}
$$

For each $k$, we remove the following vertices from $\operatorname{Vert}\left(R_{k}^{\prime}\right)$

$$
\begin{array}{lll}
x_{h_{k}+t}, x_{h_{k}+2 t+1}, \ldots, x_{h_{k}+p_{k} t+\left(p_{k}-1\right)} & \bmod n & \text { if } 1 \leq k \leq \alpha \text { and } p_{k} \neq 0 \\
x_{h_{k}+t}, x_{h_{k}+2 t+1}, \ldots, x_{h_{k}+\left(q_{k-\alpha}+1\right) t+q_{k-\alpha}} & \bmod n & \text { if } \alpha+1 \leq k \leq \alpha+\beta .
\end{array}
$$

Let $\Gamma=\left\langle R_{1}, R_{2}, \ldots R_{\alpha+\beta}\right\rangle$ be the induced subcollection on the remaining vertices of $\Sigma$, where

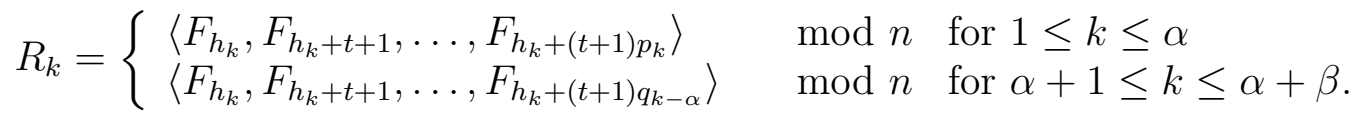

In other words, $\bmod n, \Gamma$ has facets

$$
F_{h_{1}}, F_{h_{1}+t+1}, \ldots, F_{h_{1}+(t+1) p_{1}}, F_{h_{2}}, F_{h_{2}+t+1}, \ldots, F_{h_{2}+(t+1) p_{2}}, \ldots, F_{h_{\alpha+\beta}}, \ldots, F_{h_{\alpha+\beta}+(t+1) q_{\beta}} .
$$

It is clear that each $R_{k}$ consists of runs of length one. Since $\Gamma$ is a subcollection of $\Sigma$, no runs of $R_{k}$ and $R_{k^{\prime}}$ are connected to one another if $k \neq k^{\prime}$, and hence we can conclude that $\Gamma$ is an induced subcollection of $\Delta_{t}\left(C_{n}\right)$ which is composed of runs of length one. From (7) we have the number of runs of length 1 in $\Gamma$ (or the number of facets of $\Gamma$ ) is equal to

$$
\left(p_{1}+1\right)+\left(p_{2}+1\right)+\cdots+\left(p_{\alpha}+1\right)+\left(q_{1}+1\right)+\cdots+\left(q_{\beta}+1\right)=P+Q+\alpha+\beta=i .
$$

Therefore, $\Gamma$ is an induced subcollection of $\Delta_{t}\left(C_{n}\right)$ which is composed of $i$ runs of length 1. We relabel the facets of $\Gamma$ as $\Gamma=\left\langle F_{c_{1}}, \ldots, F_{c_{i}}\right\rangle$. Now consider the following subset of $\left\{x_{c_{1}+t}, \ldots, x_{c_{i}+t}\right\}$ as $A$

$$
\bigcup_{k=1, p_{k} \neq 0}^{\alpha}\left\{x_{h_{k}+t}, x_{h_{k}+2 t+1}, \ldots, x_{h_{k}+p_{k} t+\left(p_{k}-1\right)}\right\} \cup \bigcup_{k=\alpha+1}^{\alpha+\beta}\left\{x_{h_{k}+t}, x_{h_{k}+2 t+1}, \ldots, x_{h_{k}+\left(q_{k-\alpha}+1\right) t+q_{k-\alpha}}\right\}
$$


by (6) we have:

$$
|A|=\left(p_{1}+p_{2}+\cdots+p_{\alpha}\right)+\left(q_{1}+1 \cdots+q_{\beta}+1\right)=P+Q+\beta=j .
$$

Then if we set

$$
W=\left(\bigcup_{h=1}^{i} F_{c_{h}}\right) \cup A
$$

we clearly have $\Sigma=\left(\Delta_{t}\left(C_{n}\right)\right)_{W}$. This proves the existence of $\Gamma$. We now prove its uniqueness. Let $\Lambda=\left\langle F_{s_{1}}, F_{s_{2}}, \ldots, F_{s_{i}}\right\rangle$ be an induced subcollection of $\Delta_{t}\left(C_{n}\right)$ which is composed of $i$ runs of length 1 such that $1 \leq s_{1}<s_{2}<\cdots<s_{i} \leq n$. Also let $B$ be a $j$ - subset of the set $\left\{x_{s_{1}+t}, x_{s_{2}+t}, \ldots, x_{s_{i}+t}\right\} \quad \bmod n$ such that

$$
\Sigma=\left(\Delta_{t}\left(C_{n}\right)\right) \operatorname{Vert}(\Lambda) \cup B
$$

Suppose that $\Lambda=\left\langle S_{1}, S_{2}, \ldots, S_{\alpha+\beta}\right\rangle$, such that for $k=1,2, \ldots, \alpha+\beta, S_{k}$ is an induced subcollection of $R_{k}^{\prime}$ which consists of $y_{k}$ runs of length one. By (8) we have $y_{k} \neq 0$ for all $k$. Now we prove the following claims for each $k \in\{1,2, \ldots, \alpha+\beta\}$.

(a) $F_{h_{k}} \in \Lambda$. Suppose that $1 \leq k \leq \alpha+\beta$. If $p_{k}=0$ we are clearly done, so consider the case $p_{k} \neq 0$.

Assume that $F_{h_{k}} \notin \Lambda$. Since $F_{h_{k}}$ is the only facet of $\Sigma$ which contains $x_{h_{k}}$ we can conclude that $x_{h_{k}} \notin \operatorname{Vert}(\Lambda)$. From (8), it follows that $x_{h_{k}} \in\left\{x_{s_{1}+t}, x_{s_{2}+t}, \ldots, x_{s_{i}+t}\right\}$, so

$$
x_{h_{k}}=x_{s_{a}+t} \bmod n \text { for some } a .
$$

On the other hand we know

$$
\begin{aligned}
& F_{s_{a}}=\left\{x_{s_{a}}, x_{s_{a}+1}, \ldots, x_{s_{a}+t-1}\right\} \bmod n \\
& F_{s_{a}+1}=\left\{x_{s_{a}+1}, x_{s_{a}+2}, \ldots, x_{s_{a}+t}\right\} \quad \bmod n \text {. }
\end{aligned}
$$

Since $R_{k}^{\prime}$ is an induced connected component of $\Sigma$, by (9) we can conclude that $x_{h_{k}} \in F_{s_{a}+1}$ and $F_{s_{a}}, F_{s_{a}+1} \in R_{k}^{\prime}$. However, we know $F_{h_{k}}$ is the only facet of $R_{k}^{\prime}$ which contains $x_{h_{k}}$ and so $F_{s_{a}+1}=F_{h_{k}}$ and then $s_{a}+1=h_{k} \quad \bmod n$. This and (9) imply that $t=1 \bmod n$, which contradicts our assumption $2 \leq t \leq n$.

$F_{u+t+1} \in R_{k}^{\prime}$, then $F_{u+t+1} \in S_{k}$. Assume that $F_{u+t+1} \notin S_{k}$ and $F_{u+t+1} \in R_{k}^{\prime}$. Let

$$
r_{0}=\min \left\{r: r>u, F_{r} \in S_{k} \bmod n\right\} .
$$

Since $S_{k}$ consists of runs of length one we can conclude that $r_{0} \geq u+t+1$. Since $r_{0} \neq u+t+1$ we have $r_{0} \geq u+t+2$. But then

$$
x_{u+t+1} \notin \operatorname{Vert}(\Lambda) \cup\left\{x_{s_{1}+t}, x_{s_{2}+t}, \ldots, x_{s_{i}+t}\right\}
$$

and therefore $x_{u+t+1} \notin \operatorname{Vert}(\Sigma)$ which is a contradiction.

Now for each $k$, by (a) we have $F_{h_{k}} \in \Lambda$ and from repeated applications of (b) we find that

$$
F_{h_{k}+f(t+1)} \in S_{k} \quad \text { for } f= \begin{cases}1,2, \ldots, p_{k} & 1 \leq k \leq \alpha \\ 1,2, \ldots, q_{k-\alpha} & \alpha+1 \leq k \leq \alpha+\beta .\end{cases}
$$

So $R_{k} \subseteq S_{k}$. On the other hand $S_{k}$ consists of runs of length one, so no other facet of $R_{k}^{\prime}$ can be added to it, and therefore $S_{k}=R_{k}$ for all $k$. We conclude that $\Lambda=\Gamma$ and we are therefore done. The last claim of the proposition is also apparent from this proof. 
We are now ready to compute the remaining Betti numbers.

Theorem 4.12. Let $n, i, j$ and $t$ be integers such that $n \geq 2,2 \leq t \leq n$, and $t i+j<n$. Then

$$
\beta_{i+j, t i+j}\left(R / I_{t}\left(C_{n}\right)\right)=\frac{n}{n-i t}\left(\begin{array}{l}
i \\
j
\end{array}\right)\left(\begin{array}{c}
n-i t \\
i
\end{array}\right)
$$

Proof. If $I=I_{t}\left(C_{n}\right)$, from Theorem 4.2, $\beta_{i+j, t i+j}(R / I)$ is the number of $(i+j, t i+j)$-eligible subcollections of $\Delta_{t}\left(C_{n}\right)$. Suppose that $\mathcal{R}_{(i)}$ denotes the set of all induced subcollections of $\Delta_{t}\left(C_{n}\right)$ which are composed of $i$ runs of length one. By propositions 4.10 and 4.11 there exists a one to one correspondence between the set of all $(i+j, t i+j)$-eligible subcollections of $\Delta_{t}\left(C_{n}\right)$ and the set

$$
\mathcal{R}_{(i)} \times\left(\begin{array}{c}
{[i]} \\
j
\end{array}\right)
$$

where $\left(\begin{array}{c}{[i]} \\ j\end{array}\right)$ is the set of all $j$-subsets of a set with $i$ elements. By Corollary 4.3 we have $\left|\mathcal{R}_{(i)}\right|=\beta_{i, t i}$ and since $\left|\left(\begin{array}{c}{[i]} \\ j\end{array}\right)\right|=\left(\begin{array}{l}i \\ j\end{array}\right)$ and so we apply Corollary 4.8 to observe that

$$
\beta_{i+j, t i+j}\left(R / I_{t}\left(C_{n}\right)\right)=\left(\begin{array}{l}
i \\
j
\end{array}\right) \beta_{i, t i}\left(R / I_{t}\left(C_{n}\right)\right)=\frac{n}{n-t i}\left(\begin{array}{l}
i \\
j
\end{array}\right)\left(\begin{array}{c}
n-t i \\
i
\end{array}\right) .
$$

Finally, we put together Theorem 4.2, Proposition 4.4 and [AF15, Theorem 5.1 and Theorem 2.8]. Note that the case $t=2$ is the case of graphs which appears in [Jac04. Also note that $\beta_{i, j}\left(R / I_{t}\left(C_{n}\right)\right)=0$ for all $i \geq 1$ and $j>t i$, see for example see for example [Jac04, $3.3 .4]$.

Theorem 4.13 (Betti numbers of path ideals of cycles). Let $n, t, p$ and $d$ be integers such that $n \geq 2,2 \leq t \leq n, n=(t+1) p+d$, where $p \geq 0,0 \leq d \leq t$. Then the $\mathbb{N}$-graded Betti numbers of the path ideal of the graph cycle $C_{n}$ are given by

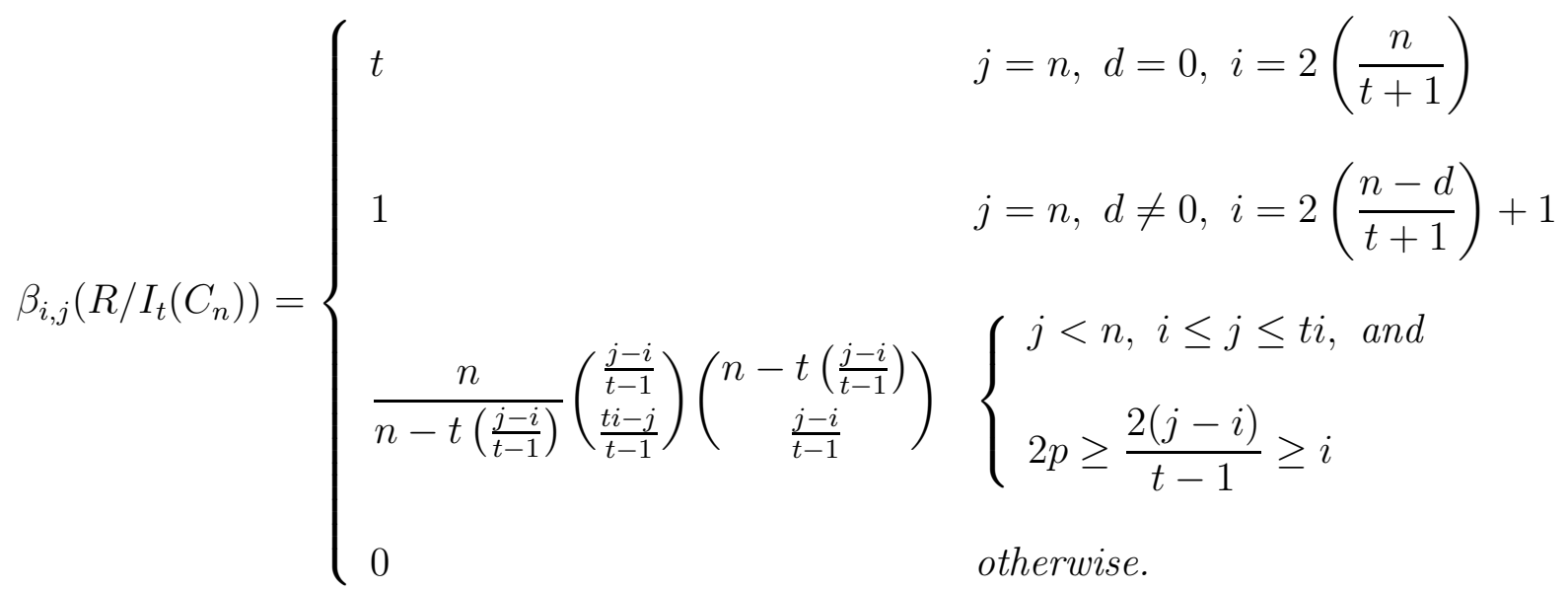

Proof. We only need to make sure we have the correct conditions for the Betti numbers to be nonzero. When $j<n, \beta_{i, j}\left(R / I_{t}\left(C_{n}\right)\right) \neq 0 \Longleftrightarrow$ 


$$
\begin{aligned}
& \Longleftrightarrow\left\{\begin{array}{l}
\frac{j-i}{t-1} \geq \frac{t i-j}{t-1} \\
n-\frac{t(j-i)}{t-1} \geq \frac{j-i}{t-1}
\end{array}\right. \\
& \Longleftrightarrow\left\{\begin{array}{l}
2 j \geq(t+1) i \\
n \geq\left(\frac{t+1}{t-1}\right)(j-i)
\end{array}\right. \\
& \Longleftrightarrow\left\{\begin{array}{l}
2 j \geq(t+1) i \\
(t+1) p+d \geq\left(\frac{t+1}{t-1}\right)(j-i)
\end{array}\right. \\
& \Longleftrightarrow\left\{\begin{array}{l}
2 j \geq(t+1) i \\
p+\frac{d}{t+1} \geq \frac{j-i}{t-1} \\
2 p \geq \frac{2(j-i)}{t-1} \geq i
\end{array}\right.
\end{aligned}
$$

Theorem 4.14 (Betti numbers of path ideals of lines ). Let $n, t, p$ and $d$ be integers such that $n \geq 2,2 \leq t \leq n, n=(t+1) p+d$, where $p \geq 0,0 \leq d \leq t$. Then the $\mathbb{N}$-graded Betti numbers of the path ideal of the path graph $L_{n}$ are nonzero and equal to

$$
\beta_{i, j}\left(R / I_{t}\left(L_{n}\right)\right)=\left(\begin{array}{c}
\frac{j-i}{t-1} \\
\frac{t i-j}{t-1}
\end{array}\right)\left(\begin{array}{c}
n-t\left(\frac{j-i}{t-1}\right) \\
\frac{j-i}{t-1}
\end{array}\right)+\left(\begin{array}{c}
\frac{j-i}{t-1}-1 \\
\frac{t i-j}{t-1}
\end{array}\right)\left(\begin{array}{c}
n-t\left(\frac{j-i}{t-1}\right) \\
\frac{j-i}{t-1}-1
\end{array}\right)
$$

if and only if

(i) $j \leq n$ and $i \leq j \leq t i$;

(ii) If $d<t$ then $p \geq \frac{j-i}{t-1} \geq i / 2$ where both inequalities cannot be $=$ at the same time;

(iii) If $d=t$ then $(p+1) \geq \frac{j-i}{t-1} \geq i / 2$ where both inequalities cannot be $=$ at the same time.

Proof. We use induction on $n$. Suppose $n=2$ so $t=2$. Then we have $I_{t}\left(L_{n}\right)=\left(x_{1} x_{2}\right)$ and it is clear that the only nonzero Betti number of $R / I_{t}\left(L_{n}\right)$ is $\beta_{1,2}=1$. So the assertion is clear. So suppose $n>2$. To continue we will use the following formula for each integer $N, R>1$

$$
\left(\begin{array}{l}
N \\
R
\end{array}\right)+\left(\begin{array}{c}
N \\
R-1
\end{array}\right)=\left(\begin{array}{c}
N+1 \\
R
\end{array}\right)
$$

From [BHK10] we have the recursive formula

$$
\beta_{i, j}\left(R / I_{t}\left(L_{n}\right)\right)=\beta_{i, j}\left(R / I_{t}\left(L_{n-1}\right)\right)+\beta_{i-1, j-t}\left(R / I_{t}\left(L_{n-(t+1)}\right)\right)+\beta_{i-2, j-t-1}\left(R / I_{t}\left(L_{n-(t+1)}\right)\right)
$$


which using the induction hypothesis leads to the following calculation for $\beta_{i, j}\left(R / I_{t}\left(L_{n}\right)\right)$

$$
\begin{aligned}
& \left(\begin{array}{c}
\frac{j-i}{t-1} \\
\frac{t i-j}{t-1}
\end{array}\right)\left(\begin{array}{c}
n-1-t\left(\frac{j-i}{t-1}\right) \\
\frac{j-i}{t-1}
\end{array}\right)+\left(\begin{array}{c}
\frac{j-i}{t-1}-1 \\
\frac{t i-j}{t-1}
\end{array}\right)\left(\begin{array}{c}
n-1-t\left(\frac{j-i}{t-1}\right) \\
\frac{j-i}{t-1}-1
\end{array}\right) \\
& +\left(\begin{array}{c}
\frac{j-t-i+1}{t-1} \\
\frac{t i-t-j+t}{t-1}
\end{array}\right)\left(\begin{array}{c}
n-(t+1)-t\left(\frac{j-t-i+1}{t-1}\right) \\
\frac{j-t-i+1}{t-1}
\end{array}\right)+\left(\begin{array}{c}
\frac{j-t-i+1}{t-1}-1 \\
\frac{t i-t-j+t}{t-1}
\end{array}\right)\left(\begin{array}{c}
n-(t+1)-t\left(\frac{j-t-i+1}{t-1}\right) \\
\frac{j-t-i+1}{t-1}-1
\end{array}\right) \\
& +\left(\begin{array}{c}
\frac{j-t-1-i+2}{t-1} \\
\frac{t i-2 t-j+t+1}{t-1}
\end{array}\right)\left(\begin{array}{c}
n-(t+1)-t\left(\frac{j-t-1-i+2}{t-1}\right) \\
\frac{j-t-1-i+2}{t-1}
\end{array}\right)+\left(\begin{array}{c}
\frac{j-t-1-i+2}{t-1}-1 \\
\frac{t i-2 t-j+t+1}{t-1}
\end{array}\right)\left(\begin{array}{c}
n-(t+1)-t\left(\frac{j-t-1-i+2}{t-1}\right) \\
\frac{j-t-1-i+2}{t-1}-1
\end{array}\right) .
\end{aligned}
$$

For ease of writing, we set $A=\frac{j-i}{t-1}$ and $B=\frac{t i-j}{t-1}$, so we have that

$$
\begin{aligned}
& \beta_{i, j}\left(R / I_{t}\left(L_{n}\right)\right)=\left(\begin{array}{c}
A \\
B
\end{array}\right)\left(\begin{array}{c}
n-1-t A \\
A
\end{array}\right)+\left(\begin{array}{c}
A-1 \\
B
\end{array}\right)\left(\begin{array}{c}
n-1-t A \\
A-1
\end{array}\right) \\
& +\left(\begin{array}{c}
A-1 \\
B
\end{array}\right)\left(\begin{array}{c}
n-1-t A \\
A-1
\end{array}\right)+\left(\begin{array}{c}
A-2 \\
B
\end{array}\right)\left(\begin{array}{c}
n-1-t A \\
A-2
\end{array}\right)+\left(\begin{array}{c}
A-1 \\
B-1
\end{array}\right)\left(\begin{array}{c}
n-1-t A \\
A-1
\end{array}\right)+\left(\begin{array}{c}
A-2 \\
B-1
\end{array}\right)\left(\begin{array}{c}
n-1-t A \\
A-2
\end{array}\right) \\
& =\left[\left(\begin{array}{c}
A-1 \\
B
\end{array}\right)+\left(\begin{array}{c}
A-1 \\
B-1
\end{array}\right)\right]\left(\begin{array}{c}
n-1-t A \\
A-1
\end{array}\right)+\left[\left(\begin{array}{c}
A-2 \\
B
\end{array}\right)+\left(\begin{array}{c}
A-2 \\
B-1
\end{array}\right)\right]\left(\begin{array}{c}
n-1-t A \\
A-2
\end{array}\right)+\left(\begin{array}{c}
A \\
B
\end{array}\right)\left(\begin{array}{c}
n-1-t A \\
A
\end{array}\right)+\left(\begin{array}{c}
A-1 \\
B
\end{array}\right)\left(\begin{array}{c}
n-1-t A \\
A-1
\end{array}\right) \\
& =\left(\begin{array}{c}
A \\
B
\end{array}\right)\left(\begin{array}{c}
n-1-t A \\
A-1
\end{array}\right)+\left(\begin{array}{c}
A-1 \\
B
\end{array}\right)\left(\begin{array}{c}
n-1-t A \\
A-2
\end{array}\right)+\left(\begin{array}{c}
A \\
B
\end{array}\right)\left(\begin{array}{c}
n-1-t A \\
A
\end{array}\right)+\left(\begin{array}{c}
A-1 \\
B
\end{array}\right)\left(\begin{array}{c}
n-1-t A \\
A-1
\end{array}\right) \\
& \left.=\left(\begin{array}{c}
A \\
B
\end{array}\right)\left[\left(\begin{array}{c}
n-1-t A \\
A-1
\end{array}\right)+\left(\begin{array}{c}
n-1-t A \\
A
\end{array}\right)\right]+\left(\begin{array}{c}
A-1 \\
B
\end{array}\right)\left[\begin{array}{c}
n-1-t A \\
A-2
\end{array}\right)+\left(\begin{array}{c}
n-1-t A \\
A-1
\end{array}\right)\right] \\
& =\left(\begin{array}{c}
A \\
B
\end{array}\right)\left(\begin{array}{c}
n-t A \\
A
\end{array}\right)+\left(\begin{array}{c}
A-1 \\
B
\end{array}\right)\left(\begin{array}{c}
n-t A \\
A-1
\end{array}\right) .
\end{aligned}
$$

Using the notation above, we see that $\beta_{i, j}\left(R / I_{t}\left(L_{n}\right)\right) \neq 0$ if and only if

$$
[B \leq A \text { and } A \leq n-t A] \text { or }[B \leq A-1 \text { and } A-1 \leq n-t A]
$$

which is equivalent to saying that

$$
B \leq A \text { and } A-1 \leq n-t A \text { where both } \geq \text { cannot be }=\text { at the same time. }
$$

In other words $\beta_{i, j}\left(R / I_{t}\left(L_{n}\right)\right) \neq 0$ if and only if

$$
\begin{array}{ll}
\frac{j-i}{t-1} \geq \frac{t i-j}{t-1} \text { and } n-\frac{t(j-i)}{t-1} \geq \frac{j-i}{t-1}-1 & \Longleftrightarrow \\
2 j \geq(t+1) i \text { and } n+1 \geq\left(\frac{t+1}{t-1}\right)(j-i) & \Longleftrightarrow \\
2 j \geq(t+1) i \text { and }(t+1) p+d+1 \geq\left(\frac{t+1}{t-1}\right)(j-i) & \Longleftrightarrow \\
2 j \geq(t+1) i \text { and } p+\frac{d+1}{t+1} \geq \frac{j-i}{t-1} &
\end{array}
$$

where in each line both $\geq$ cannot be $=$ at the same time. This is equivalent to

$$
\begin{cases}2 p \geq \frac{2(j-i)}{t-1} \geq i & \text { if } d<t \\ 2(p+1) \geq \frac{2(j-i)}{t-1} \geq i & \text { if } d=t\end{cases}
$$


(note that $\frac{j-i}{t-1}$ from Theorem 4.2 and Definition 4.1 is an integer) where both $\geq$ cannot be $=$ at the same time in the second line.

We can now easily derive the projective dimension and regularity of path ideals of paths, which were known before. The projective dimension of paths (Part i below) was computed in [HT10] using different methods. The case $t=2$ is the case of graphs which appears in [Jac04]. Part ii of the following Corollary reproves [BHK10, Theorem 5.3] which computes the Castelnuovo-Mumford regularity of path ideal of a path. The case of cycles was done in AF15.

Corollary 4.15 (Projective dimension and regularity of path ideals of paths). Let $n, t, p$ and $d$ be integers such that $n \geq 2,2 \leq t \leq n, n=(t+1) p+d$, where $p \geq 0,0 \leq d \leq t$. Then

(i) The projective dimension of the path ideal of a path $L_{n}$ is given by

$$
p d\left(R / I_{t}\left(L_{n}\right)\right)= \begin{cases}2 p & d \neq t \\ 2 p+1 & d=t\end{cases}
$$

(ii) The regularity of the path ideal of a path $L_{n}$ is given by

$$
\operatorname{reg}\left(R / I_{t}\left(L_{n}\right)\right)= \begin{cases}p(t-1) & d<t \\ (p+1)(t-1) & d=t\end{cases}
$$

Proof. $\quad$ (i) By using Theorem 4.13 we know that if $\beta_{i, j}\left(R / I_{t}\left(L_{n}\right) \neq 0\right.$ then $i \leq 2 p+1$ when $d=t$ and therefore $p d\left(R / I_{t}\left(L_{n}\right)\right) \leq 2 p+1$. On the other hand by applying Theorem 4.13 we have

$$
\beta_{2 p+1, n}\left(R / I_{t}\left(L_{n}\right)\right)=\left(\begin{array}{c}
p+1 \\
p
\end{array}\right)\left(\begin{array}{c}
p \\
p+1
\end{array}\right)+\left(\begin{array}{l}
p \\
p
\end{array}\right)\left(\begin{array}{l}
p \\
p
\end{array}\right)=1 \neq 0 .
$$

Then we can conclude that $p d\left(R / I_{t}\left(L_{n}\right)\right)=2 p+1$. Now we suppose that $d \neq t$. From Theorem 4.14 we can see that if $\beta_{i, j}\left(R / I_{t}\left(L_{n}\right)\right) \neq 0$ then $2 p \geq i$ and therefore $p d\left(R / I_{t}\left(L_{n}\right)\right) \leq 2 p$. On the other hand, by applying Theorem 4.14, we can see that

$$
\beta_{2 p, p(t+1)}\left(R / I_{t}\left(L_{n}\right)\right)=\left(\begin{array}{c}
p \\
p
\end{array}\right)\left(\begin{array}{c}
p+d \\
p
\end{array}\right)+\left(\begin{array}{c}
p-1 \\
p
\end{array}\right)\left(\begin{array}{c}
p \\
p
\end{array}\right)=\left(\begin{array}{c}
p+d \\
p
\end{array}\right) \neq 0 .
$$

Therefore $p d\left(R / I_{t}\left(L_{n}\right)\right) \geq 2 p$ and we have $p d\left(R / I_{t}\left(L_{n}\right)\right)=2 p$.

(ii) By definition, the regularity of a module $M$ is $\max \left\{j-i \mid \beta_{i, j}(M) \neq 0\right\}$. By Theorem 4.14, we know exactly when the graded Betti numbers of $R / I_{t}\left(L_{n}\right)$ are nonzero, and the formula follows directly.

\section{ACKNOWLEDGEMENT}

We gratefully acknowledge the helpful computer algebra systems CoCoA ABLre and Macaulay2 GSre, without which our work would have been difficult or impossible. We also thank the referee whose comments improved the paper. 


\section{REFERENCES}

[ABLre] J. Abbott, A. M. Bigatti, and G. Lagorio. CoCoA-5: a system for doing Computations in Commutative Algebra. Available at http://cocoa.dima.unige.it, software.

[AF15] A. Alilooee and S. Faridi. On the resolution of path ideals of cycles. Comm. Algebra, 43:5413-5433, 2015 .

[Ali15] A. Alilooee. Algebraic Properties of Monomial Ideals. PhD thesis, Dalhousie University, 2015.

[BHK10] R. Bouchat, H. T. Ha, and A. O' Keefe. Path ideals of rooted trees and their graded betti numbers. J. Combinatorial Theory, Ser. A, 118:2411-2425, 2010.

[CN99] A. Conca and E. De Negri. M-sequences, graph ideals and ladder ideals of linear types. J. Algebra, 211:599-624, 1999.

[Gri93] Ralph Grimaldi. Discrete and combinatorial mathematics - an applied introduction (3. ed.). Addison-Wesley, 1993.

[GSre] D. R. Grayson and M. E. Stillman. Macaulay 2, a software system for research in algebraic geometry. Available http://www.math.uiuc.edu/Macaulay2/, software.

[HT10] J. He and A. Van Tuyl. Algebraic properties of path ideal of a tree. Comm. Algebra, 38:1725-1742, 2010.

[Jac04] S. Jacques. Betti Numbers of Graph Ideals. PhD thesis, The University of Sheffield, arXiv.math.AC/0410107, 2004.

[Sta12] R.P. Stanley. Enumerative combinatorics. Volume 1. Cambridge Studies in Advanced Mathematics. Cambridge University Press, Cambridge, second edition, 2012.

University of Wisconsin-Stout, Department of Mathematics and Statistics, Jarvis HallScience Wing, Menomonie, WI, USA

E-mail address: a-alilooeedolatabad@wiu.edu

Dalhousie University, Department of Mathematics, 6316 Coburg Rd. Halifax, ns, Canada B3H 4R2

E-mail address: faridi@mathstat.dal.ca 